\title{
Developmental Trajectory of Object Recognition Memory in Infant Rhesus Macaques with and without Neonatal Hippocampal Lesions
}

\author{
Alyson Zeamer, ${ }^{1}$ Eric Heuer, ${ }^{2}$ and Jocelyne Bachevalier ${ }^{2}$ \\ ${ }^{1}$ Department of Pathology, Anatomy, and Cell Biology, Thomas Jefferson University, Philadelphia, Pennsylvania 19107, and ${ }^{2}$ Yerkes National Primate \\ Research Center and Department of Psychology, Emory University, Atlanta, Georgia 30329
}

To examine the developmental trajectory of object recognition memory and its neural substrate, 10-12-d-old monkeys (Macaca mulatta) received sham operations or neurotoxic hippocampal lesions and were tested at the ages of 1.5, 6, and 18 months on the visual paired-comparison task using delays of 10,30,60, and $120 \mathrm{~s}$. In sham-operated controls, incidental recognition memory was present at 1.5 months, became more robust at 6 months, and was delay-dependent by 18 months of age, suggesting that the brain structures mediating these early developing recognition abilities may undergo significant modifications after 6 months of age in monkeys. A similar developmental progression was also observed in animals with neonatal hippocampal lesions, although the delay-dependent effect at 18 months was significantly more pronounced after the neonatal hippocampal lesions, suggesting that with maturation animals with neonatal hippocampal lesions grow into a recognitionmemory deficit. These findings suggest not only that the medial temporal cortical areas, known to mediate incidental recognition memory processes in adulthood, could support these processes in early infancy even when long delays are used, but also that later in development, after reaching functional maturity, the hippocampus begins to interact with the medial temporal cortical areas to mediate this function.

\section{Introduction}

Due to its incidental nature, the visual paired-comparison (VPC) task has been the task of choice to study recognition-memory development in primates, including humans (Fagan, 1970). In this task, recognition memory is indexed by longer looking time to novel stimuli. In humans, this recognition-memory ability is present as early as 3-4 d of age with either no delays or $2 \mathrm{~min}$ delays (Pascalis and de Schonen, 1994) and 3-month-old infants show strong novelty preference even at $24 \mathrm{~h}$ delays (Pascalis et al., 1998). By 4 weeks of age, infant macaques show novelty preference and this preference becomes stronger by 13 weeks of age (Gunderson and Sackett, 1984, Bachevalier et al., 1993). In addition, by 6 weeks of age, novelty preference is present after long delays of $24 \mathrm{~h}$ (Gunderson and Swartz, 1984). Although these studies demonstrate the presence of robust recognition-memory abilities in infancy, the brain structures that could support this early developing memory ability are still poorly understood. Because performance on this task is altered in a delay-dependent manner by selective hippocampal damage in adult monkeys and

Received Jan. 4, 2010; revised April 21, 2010; accepted May 10, 2010.

This work was supported by grants from the National Institute of Mental Health (MH-58846), the Yerkes Base Grant NIH RR00165, and the Center for Behavioral Neuroscience Grant NSF IBN-9876754. We thank the University of Texas Health Science Center at Houston veterinary and animal husbandry staff for expert animal care, Belinda Rivera for the care and handling of animals during the MR imaging procedures, Edward F. Jackson for assistance in neuroimaging techniques, Michelline Resende for help with the training of the animals, and Eugena Mitchell and Andy Kazama for help with reliability measurements.

Correspondence should be addressed to Alyson Zeamer, Thomas Jefferson University, 1020 Locust JAH 511, Philadelphia, PA 19107. E-mail: alyson.zeamer@jefferson.edu.

DOI:10.1523/JNEUROSCI.0022-10.2010

Copyright $\odot 2010$ the authors $\quad 0270-6474 / 10 / 309157-09 \$ 15.00 / 0$ humans (McKee and Squire, 1993; Zola et al., 2000; Nemanic et al., 2004; Pascalis et al., 2004), it has been argued that the presence of incidental recognition memory in early infancy may be supported by an early functional maturation of the hippocampus (Diamond, 1990; Nelson, 1995; Nelson and Webb, 2003). However, direct evidence to support this assertion is still lacking. Only two developmental studies in monkeys have assessed the effects of neonatal ( $8-10 \mathrm{~d}$ of age) lesions to the hippocampal formation on the development of novelty preference. In the first study (Bachevalier et al., 1993), neonatal damage to the medial temporal lobe abolished novelty preference at 15 and $30 \mathrm{~d}$ of age even at short delays of $10 \mathrm{~s}$. In the second study (Pascalis and Bachevalier, 1999), adult monkeys that had received neonatal hippocampal lesions showed a delay-dependent recognition-memory deficit occurring only at delays lasting $30 \mathrm{~s}$ and longer. However, in both developmental studies the lesions were not restricted to the hippocampus, but involved the amygdala and/or the adjacent cortical areas (perirhinal and parahippocampal cortical areas). Given that selective lesions to either of these cortical areas in adult monkeys also abolish novelty preference, albeit at different delays (Buffalo et al., 1999; Nemanic et al., 2004), it is thus possible that recognition-memory deficits seen after neonatal hippocampal lesions resulted either from combined lesions of the hippocampus and temporal cortical areas or from damage to the temporal cortical structures alone.

The goal of the present study was twofold. Given the lack of longitudinal studies that have examined the normal development of incidental recognition-memory abilities, the first aim was to systematically follow performance of infant monkeys on the VPC task from 1.5 to 18 months using delays varying from 10 to $120 \mathrm{~s}$. 
The second aim was to assess the effects of selective neonatal hippocampal lesions on the development of incidental recognition-memory processes using the same maturational points and delays. Preliminary reports of the findings were published in abstract form (Resende et al., 2002; Zeamer et al., 2006).

\section{Materials and Methods}

All experimental procedures were approved by the Institutional Animal Care and Use Committee of the University of Texas at Houston where this study began and by Emory University where it was completed. All rearing and behavioral testing procedures were kept constant between the two institutions.

\section{Subjects}

The subjects were 11 full-term infant rhesus monkeys (Macaca mulatta), 6 males and 5 females, acquired from the MD Anderson Cancer Center Science Park breeding facility. Between 1 and $4 \mathrm{~d}$ after birth, they were brought from MD Anderson Cancer Center Science Park (Bastrop, TX) to the primate nursery at MD Anderson Cancer Center (Houston, TX) where they were raised. They were nursery reared according to procedures developed by Sackett and colleagues (2002) that allow normal growth as well as the development of species-specific social skills. These procedures included daily social interactions with peers, intensive human contact, and cognitive testing that began in the first weeks of life and continued through adolescence [(for additional details on rearing conditions, see Goursaud and Bachevalier (2007)]. They were first handfed a diet of infant Similac formula (Abbot Laboratories) and then, starting around 8 months of age, fed jumbo primate chow (Lab Diet 5037; PMI Nutrition International) and fresh fruit daily. At 10-12 d, three males and three females received a sham operation (group Neo-C), and three males and two females received MRI-guided neurotoxic lesions of the hippocampus (group Neo-Hibo), bilaterally. All animals received their behavioral testing at the University of Texas Medical Center at Houston, except for two monkeys in each group that received the testing at 18 months of age after they were moved to Yerkes National Primate Research Center (Atlanta, GA).

\section{Surgical procedures}

Presurgical and postsurgical magnetic resonance imaging scans. Two neuroimaging sessions were performed (Málková et al., 2001; Nemanic et al., 2002), one immediately before surgery on all 11 animals and the other 5-8 d postsurgery only on animals of group Neo-Hibo. For each neuroimaging session, the subjects were placed in an induction box saturated with isoflurane gas, intubated, and maintained under isoflurane gas $(1.0-3.0 \%, v / v$, to effect) throughout the procedure. They were moved to the neuroimaging suite where they were placed in the stereotaxic apparatus and positioned at the center of a GE Signa 1.5 tesla echo speed scanner (GE Medical Systems). All images were acquired with a $5 \mathrm{~cm}$ surface coil. A short sagittal scout [T1-weighted spin-echo sequence, echo time $(\mathrm{TE})=11 \mathrm{~ms}$, repetition time $(\mathrm{TR})=450 \mathrm{~ms}$, contiguous 4 $\mathrm{mm}$ sections, $12 \mathrm{~cm}$ field of view (FOV), $256 \times 256$ matrix] was used to align two MR sequences in the coronal plane. The first was a threedimensional, T1-weighted, fast-spoiled gradient-echo sequence $(\mathrm{TE}=$ $2.6 \mathrm{~ms}, \mathrm{TR}=10.2 \mathrm{~ms}, 25^{\circ}$ flip angle, contiguous $1 \mathrm{~mm}$ sections, $12 \mathrm{~cm}$ FOV, $256 \times 256$ matrix) used to calculate the coordinates for each injection site along the entire length of the hippocampal formation. The second was a set of three fluid-attenuated inversion-recovery (FLAIR) sequences $[\mathrm{TE}=140 \mathrm{~ms}, \mathrm{TR}=10,000 \mathrm{~ms}$, inversion time $=2200 \mathrm{~ms}$, contiguous $3 \mathrm{~mm}$ sections, $12 \mathrm{~cm}$ FOV, $256 \times 256$ matrix], offset by $1 \mathrm{~mm}$ posteriorly, and used to identify the location of hypersignals around the injection sites indicative of edema caused by neurotoxin-induced cell death. These hypersignals were used to estimate the extent of lesions. An additional magnetic resonance imaging (MRI) session, using T1 high-resolution MRI images, done approximately one year after surgery in animals of group NeoHibo, was also used to estimate the percentage reduction of the hippocampal formation bilaterally (Nemanic et al., 2002).

Surgery. Immediately after the MRI procedures, the animals were kept anesthetized in the stereotaxic apparatus and moved to the surgical suite. All surgical procedures were performed under deep anesthesia using aseptic techniques. Throughout surgery, the animal was maintained on isoflurane gas $(1.0-2.0 \%, \mathrm{v} / \mathrm{v}$, to effect), an IV drip containing dextrose and $0.45 \%$ sodium chloride was used to maintain normal hydration, a heating pad was placed under the animal to prevent hypothermia, and vital signs (heart and respiration rates, expired $\mathrm{CO}_{2}$, and temperature) were monitored until the monkey fully recovered from anesthesia.

The scalp was shaved, the skin disinfected with Nolvasan solution, and a long-lasting local anesthetic (Marcaine 25\%, $1.5 \mathrm{ml}$ ) was injected subcutaneously along the incision line. The skin was cut longitudinally from the occiput to the midorbital ridge, connective tissue was gently retracted, and two craniotomies were performed bilaterally above the injection sites. Bone wax (2.5 g; Ethicon) was applied to prevent excessive bleeding from the bone and the dura was opened. Two Hamilton syringes filled with ibotenic acid (10 mg/ml in PBS, pH 7.4; Biosearch Technologies) and held by Kopf electrode manipulators (David Kopf Instruments) were lowered simultaneously at each injection site in the hippocampus of each hemisphere. At the level of the uncus, two injections were placed 4 $\mathrm{mm}$ apart in the mediolateral plane and posteriorly, five injections were placed $2 \mathrm{~mm}$ apart along the body of the hippocampus. A total of $5.0 \mu \mathrm{l}$ of ibotenic acid were injected bilaterally and for each site the drug was injected at a rate of $0.4 \mu \mathrm{l}$ of per min, followed by a 3 min delay before retracting the needle to permit diffusion of the neurotoxin and minimize its spread along the needle track. Once all injections were completed, the wound was closed in anatomical layers, the anesthetic was withdrawn, and the animal recovered in the surgical suite until it could breathe on its own. The animal was then moved back to the nursery and placed in an incubator ventilated with oxygen until the next day. Beginning $12 \mathrm{~h}$ before surgery and lasting $7 \mathrm{~d}$ after surgery, dexamethasone sodium phosphate $(0.4 \mathrm{mg} / \mathrm{kg}$, i.m. ) and cephazolin $(25 \mathrm{mg} / \mathrm{kg}$, i.m. $)$ were given to reduce edema and prevent infection, respectively. In addition, acetaminophen $(10 \mathrm{mg} / \mathrm{kg}$, p.o.) was given four times a day for $3 \mathrm{~d}$ after surgery for relief of pain.

The sham lesions followed the same procedures, except that the injection needles were not lowered within the brain. Preoperative and postoperative treatment was also identical.

\section{MRI-based lesion evaluation}

Because all subjects are still participating in other cognitive experiments, no histological evaluations are available. Therefore, estimation of lesion extent is provided using the FLAIR images obtained 1 week postsurgery and the T1 high-resolution images obtained one year postsurgery (for review, see Málková et al., 2001; Nemanic et al., 2002). Briefly, the hypersignals on the FLAIR images acquired at $1 \mathrm{~mm}$ intervals were visually identified and plotted onto corresponding coronal sections of a 1-weekold normal rhesus monkey brain (J. Bachevalier, unpublished data). These images were then imported into ImageJ to measure the surface area (in square pixels) of hypersignals within the left and right hippocampal formation (including all CA fields, dentate gyrus, and subicular complex) as well as adjacent neural structures (i.e., perirhinal cortex, entorhinal cortex, areas TH and TF on the parahippocampal gyrus, and amygdala), if any. Estimated percentage of hippocampal volume damaged was then calculated by dividing the total volume of hypersignals for the right and left hippocampus by the normal hippocampal volume obtained from the normal 1-week-old monkey brain. In addition, to estimate the percentage reduction of hippocampal volume one year after surgery, each T1 image at $1 \mathrm{~mm}$ intervals throughout the entire hippocampus was imported into ImageJ and the surface area of the hippocampus on each image was measured (in square pixels). Percentage of reduction was then calculated using the following formula: $[100-($ total $\mathrm{H}$ volume remaining)/ (average $\mathrm{H}$ volume in normal one-year-old monkey) $\times 100]$.

\section{Behavioral procedures}

Apparatus and stimuli. Testing was conducted in a sound-attenuated room equipped with a white noise generator to reduce external noise. A 19 " monitor was positioned on a table at the animal's eye level and a video camera (Sony Digital8 TRV-140) was mounted above the screen and positioned to capture the monkey's eye movements. The camera output was fed into a time/date generator connected to a VCR (JVC HR-S4800U) that recorded eye movements for each trial and into a TV 


\section{Familiarization Phase}
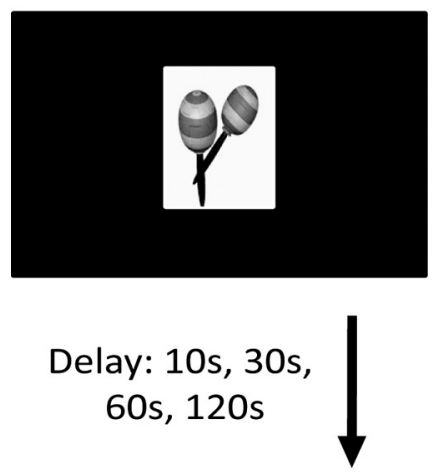

\section{Retention Test}

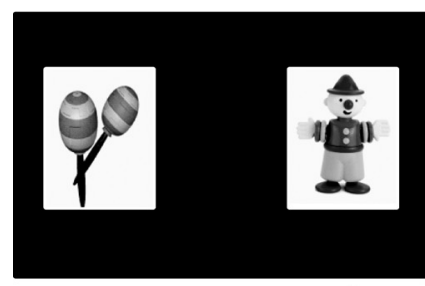

Delay: $5 \mathrm{~s}$

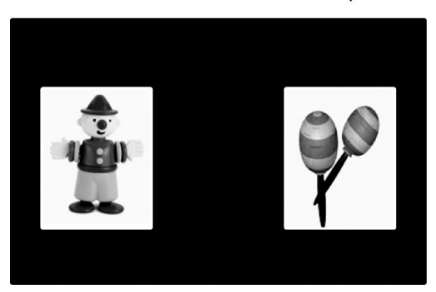

Figure 1. Example of a VPC trial. Subjects were familiarized to a picture of an object for a cumulated 30 s. Following a delay of $10,30,60$, or 120 s, they were given two 5 s retention tests separated by a $5 \mathrm{~s}$ delay, during which the now familiar picture was paired with a novel one. The left/right positions of the familiar and novel pictures were interchanged from the first to the second retention tests. The measure of recognition memory was the animal's preference to look longer at the novel picture during the retention tests.

screen to monitor the animal's looking behavior during the task. Stimuli were color pictures of variegated objects differing in color, shape, and texture (trees, animals, tools, etc.) selected from a pool of 800,000 clipart images (Nova Art Explosion 800,000 Clip Art). Stimuli were chosen so that within each pair they were similar in both size and brightness but varied widely in shape, texture, and color. However, familiar and novel stimuli were not counterbalanced across ages or delays. All images were delivered to the monitor via a computer controlled by the experimenter.

Pretraining. At 1.5 and 6 months of age, the infant was held by an experimenter $\sim 40 \mathrm{~cm}$ in front of the TV monitor. At 18 months of age, the monkey was seated in a custom made Plexiglas primate chair (University of Texas Machine Shop) that fit the animal's size. For $3 \mathrm{~d}$ before the start of formal testing, the 1.5-month-old infants were brought into the testing room where they watched a Disney cartoon for an increasing period of time each day $(15,20$, and $25 \mathrm{~min})$. A 20 min pretraining session was given for only one day when the animals reached 6 months of age. At 18 months of age, the animals were first trained over a period of a few days to enter and acclimate to the primate chair, and were then brought into the testing apparatus for $1 \mathrm{~d}$ to view a Disney cartoon. The animals were neither food deprived nor water restricted and remained in the testing apparatus for no more than $45 \mathrm{~min}$ a day.

VPC task. All monkeys were tested on the VPC task at $\sim 1.5$ (range, 1.5-1.7), $\sim 6$ (range, $6-6.5$ ), and $\sim 18$ (range, 18-18.5) months of age. Each VPC trial used new pictures and was divided into a familiarization phase and a retention phase (Fig. 1). In the familiarization phase, a stim- ulus appeared and remained in the center of the screen until the monkey spent a cumulative $30 \mathrm{~s}$ looking at the sample image as estimated online by the experimenter. After a delay of $10,30,60$, or $120 \mathrm{~s}$, which was intermixed across each testing session, two $5 \mathrm{~s}$ retention tests separated by a $5 \mathrm{~s}$ delay were given. During these two retention tests, the sample picture and a novel picture were displayed side-by-side $12 \mathrm{~cm}$ apart on the screen for $5 \mathrm{~s}$ after the animal initially looked at one of the two pictures. The left/right position of the two pictures was reversed during the two retention tests, and on the first retention test, the left/right position of the novel picture varied pseudorandomly. The screen remained black during all delay periods, and at each age, 10 trials were given at each delay with a $30 \mathrm{~s}$ intertrial interval (ITI). The animals were given $6-10$ trials per day and did not receive food treats during the testing period at 1.5 and 6 months of age, but were given mini-M\&Ms during random ITIs at 18 months to encourage them to remain focused on the screen.

\section{Data analysis}

For each trial, the video recordings of the two retention tests were analyzed frame-by-frame to calculate the amount of time the animal spent looking toward the familiar and the novel pictures (Pascalis and Bachevalier, 1999). Two observers, each blind to the position of the novel image and to the lesion group, scored the videotapes independently (interobserver reliability: Pearson $r=0.925)$. The length of time it took the animal to familiarize with the first picture (total familiarization phase) and total time looking at both pictures during the retention tests (total retention time) were recorded and analyzed. The percentage of time looking to the novel picture across the two retention tests (percent novelty) was also calculated [(time looking at novel)/ (total retention time) $\times 100$ ]. Trials for which the total looking time during the two retention tests was less than $1 \mathrm{~s}$ were rejected. Only one or two trials per animal met this criterion.

\section{Statistical analysis}

All statistical analyses were performed using the SPSS 12.0 software. A general linear model ANOVA was conducted on the three task parameters (total familiarization phase, total retention time, and percent novelty). Developmental progression of object recognition was first investigated on animals in group Neo-C using one-way ANOVAs with repeated measure for the age effect. To determine the effects of hippocampal lesion on the development of object recognition, two-way ANOVAs with a between-subject comparison for the group effect (group Neo-Hibo and group Neo-C) and within-subject comparisons using repeated measures for the delay and age effects were used. When sphericity was not assumed, a Huynh-Feldt correction was used. Post hoc Tukey tests were conducted when group differences reached significance and paired $t$ tests were used to compare performance between delays. Additionally, when interactions between factors were not significant, planned comparisons were performed between the control group and the experimental group, using one-sided planned comparisons (Pedhazur, 1982), since this comparison provides more statistical power against type II error, i.e., not rejecting the null hypothesis when it is false. Finally, onesample $t$ tests were used to evaluate group differences from chance.

\section{Results}

\section{Evaluation of hippocampal lesion}

Estimates of lesion extent from postsurgical FLAIR images for all five animals of group Neo-Hibo ranged from 33.2 to $87.4 \%$ (average, 56.9\%) (Table 1) and the percentage of volume reduction of the hippocampal formation based on one-year post T1 images ranged from 19.1 to $67.0 \%$ (average, $48.3 \%$ ) (Table 2). In Figure 2 , the extent of hypersignals seen on the postsurgical FLAIR images for each case are plotted on matched coronal sections through a normal 1-week-old infant brain. A representative case (NeoHibo-2) depicting the extent of hypersignals on FLAIR images obtained 1 week postsurgery and hippocampal volume reduction seen on T1 images 1 year postsurgery is illustrated in Figure 3.

Two cases (Neo-Hibo-2 and -3) received extensive bilateral lesions, with more extensive damage on the right than on the left (Fig. 2 and Table 1), resulting in $>50 \%$ overall volume reduction 
Table 1. Percent of intended and unintended damage

\begin{tabular}{|c|c|c|c|c|c|c|c|c|c|c|c|c|}
\hline \multirow[b]{3}{*}{ Cases } & \multirow{2}{*}{\multicolumn{4}{|c|}{$\begin{array}{l}\text { Intended damage } \\
\text { Hippocampal formation }\end{array}$}} & \multicolumn{8}{|c|}{ Unintended damage } \\
\hline & & & & & \multicolumn{4}{|c|}{ Amygdala } & \multicolumn{4}{|c|}{$\mathrm{TH} / \mathrm{TF}$} \\
\hline & $\mathrm{L} \%$ & $\mathrm{R} \%$ & $X \%$ & W\% & $\mathrm{L} \%$ & $\mathrm{R} \%$ & $X \%$ & W\% & $L \%$ & $\mathrm{R} \%$ & $\mathrm{X} \%$ & W\% \\
\hline Neo-Hibo-1 & 63.8 & 2.9 & 33.2 & 1.9 & 14.0 & 0.0 & 7.0 & 0.0 & 3.1 & 0.5 & 1.8 & 0.0 \\
\hline Neo-Hibo-2 & 54.4 & 80.9 & 67.6 & 44.0 & 0.0 & 0.0 & 0.0 & 0.0 & 21.4 & 2.7 & 12.1 & 0.6 \\
\hline Neo-Hibo-3 & 78.5 & 96.3 & 87.4 & 75.6 & 1.7 & 0.0 & 0.8 & 0.0 & 6.1 & 5.5 & 5.8 & 0.3 \\
\hline Neo-Hibo-4 & 20.3 & 67.3 & 43.8 & 13.7 & 0.0 & 4.7 & 2.4 & 0.0 & 15.3 & 0.0 & 7.6 & 0.0 \\
\hline Neo-Hibo-5 & 20.7 & 84.0 & 52.6 & 17.4 & 0.0 & 4.9 & 2.4 & 0.0 & 6.1 & 4.0 & 5.1 & 0.2 \\
\hline Average & 47.5 & 66.3 & 56.9 & 30.5 & 3.1 & 1.9 & 2.5 & 0.0 & 10.4 & 2.5 & 6.5 & 0.2 \\
\hline
\end{tabular}

Percentage of damage to the hippocampal formation for the five subjects in group Neo-Hibo, as estimated from presurgery and postsurgery coronal MR images. L\%, Percentage of damage to the left hemisphere; R\%, percentage of damage to the right hemisphere; $X \%$, average damage to both hemispheres; W\%, weighted average damage to both hemispheres $[\mathrm{W} \%=(\mathrm{L} \% \times \mathrm{R} \%) / 100]$; TH/TF, cytoarchitectonic fields of the parahippocampal gyrus, as defined by von Bonin and Bailey (1947).

Table 2. Percentage of volume reduction measured at 1 year of age

\begin{tabular}{llll}
\hline & \multicolumn{2}{l}{$\%$ Volume Reduction } & \\
\cline { 2 - 4 } Cases & $\mathrm{L} \%$ & $\mathrm{R} \%$ & $\mathrm{X} \%$ \\
\hline Ne0-Hibo-1 & 27.6 & 10.7 & 19.1 \\
Ne0-Hibo-2 & 61.2 & 72.9 & 67.0 \\
Ne0-Hibo-3 & 54.7 & 47.8 & 51.3 \\
Ne0-Hibo-4 & 33.6 & 61.7 & 47.6 \\
Ne0-Hibo-5 & 49.2 & 64.0 & 56.6 \\
Average & 45.3 & 51.4 & 48.3 \\
\hline
\end{tabular}

Percentage of hippocampal formation volume reduction and sparing for the five subjects in group Neo-Hibo, as estimated from 1 year postsurgical coronal MR images. L\%, Percentage of damage to the left hemisphere; R\%, percentage of damage to the right hemisphere; $X \%$, average damage to both hemispheres.

of the hippocampus (Table 2). For both cases, sparing mostly occurred in the left uncus and in the medial part of the caudalmost portion of the left hippocampus (Fig. 2, levels 0 to -9 ). The extent of hippocampal lesions in the three remaining cases (NeoHibo-1, 4 and 5) was more unilateral, with extensive damage on one side $(>60 \%)$ but moderate to mild damage on the other $(<21 \%)$. Cases Neo-Hibo-1 and -4 had sparing along almost the entire length of one hippocampus, since the neurotoxic injections reached the most lateral part of the hippocampus (Fig. 2, levels 0 to -9 on the right for Neo-Hibo-1 and on the left for Neo-Hibo-4). For case Neo-Hibo-5, damage on the left was located in the uncus and lateral portions of the hippocampus (Fig. 2 , levels 0 and -6$)$. It is important to note that, although the volume of damage to the left hippocampus was mild in both cases Neo-Hibo-4 and -5, the damage included the CA2 and CA1 fields of the hippocampus. This mild damage most likely disrupted the normal functioning of the trisynaptic hippocampal circuit as well as the direct entorhinal-CA1 pathway. For all cases, unintended damage to adjacent structures was minimal and restricted to $6.5 \%$ damage to areas $\mathrm{TH} / \mathrm{TF}$ and $2.5 \%$ damage to the amygdala (Table 1 ).

\section{Development of object recognition memory}

Data for the sham-operated controls were first analyzed to assess the development of object recognition memory. As shown in Table 3 and Figure 4, novelty preference remained significantly above chance level at all ages and delays but varied across ages. Thus, performance across the four delays became stronger from 1.5 to 6 months of age, and remained stable until 18 months, as revealed by a significant age effect $\left(F_{(2,10)}=4.41, p<0.05\right)$ but no delay effect $\left[F_{(3,15)}=1.24\right.$, not significant (ns) $]$ and no age $\times$ delay interaction $\left(F_{(3,60)}=1.83\right.$, ns). Overall, novelty preference was significantly more robust at 6 months than at 1.5 months $(p<0.001)$. Separate ANOVAs at each age revealed no delay effect at 1.5 and 6 months $\left(F_{(3,15)}=0.902\right.$, ns; and $F_{(3,15)}=1.04$, $\mathrm{ns}$, respectively), whereas this effect reached significance at 18 months $\left(F_{(3,15)}=3.79, p=0.03\right)$. At this age, paired-sample $t$ tests revealed stronger novelty preference at 10 and $30 \mathrm{~s}$ delays than at $120 \mathrm{~s}$ delay ( $p=0.059$ and $p=0.046$, respectively), indicating the presence of a delay-dependent effect on novelty preference only at 18 months of age. This effect cannot be attributed to changes in viewing behaviors of the animals across the delays since at all three ages, total familiarization time or total retention time did not differ as a function of age or delay $(p>$ 0.05 for all).

\section{Effects of neonatal hippocampal lesion}

Novelty preference in group Neo-Hibo followed a developmental pattern similar to that of group Neo-C (Table 3 and Fig. 5). Thus, novelty preference in group Neo-Hibo also increased significantly with age $\left(F_{(2,8)}=14.83, p<0.002\right)$. Overall, novelty preference across delays was more robust at 6 months than at 1.5 months $(p<0.001)$. In addition, the interaction between age and delay was also significant $\left(F_{(6,24)}=4.15, p<0.005\right)$. As shown in Figure 5, this interaction demonstrated that, whereas novelty preference in group Neo-Hibo did not vary across delays at the two youngest ages $\left(F_{(3,12)}=0.49\right.$, ns; and $F_{(3,12)}=2.24$, ns, respectively), it did at 18 months of age $\left(F_{(3,12)}=9.58, p=0.002\right)$. Post hoc analysis of the data at the oldest age revealed that novelty preference was more robust at delays of 10,30 , and $60 \mathrm{~s}$ than at delays of $120 \mathrm{~s}$ ( $p<0.04,0.03$, and 0.003 , respectively).

Direct comparisons with group Neo-C revealed no overall group effect $\left(F_{(1,9)}=1.98\right.$, ns), but a significant age effect $\left(F_{(2,18)}=13.25\right.$, $p<0.001)$ and a marginal delay effect $\left(F_{(3,27)}=2.65, p=0.07\right)$. None of the interactions between the factors reached significance, except for the age $\times$ delay interaction $\left(F_{(6,54)}=5.06, p<0.001\right)$. As shown in Figure 5, whereas novelty preference scores were similar in both groups and for all delays at the ages of 1.5 and 6 months $\left(F_{(3,12)}=0.49\right.$, ns; and $F_{(3,12)}=2.28$, ns, respectively $)$, there was a significant group $\times$ delay interaction $\left(F_{(3,27)}=3.68, p=0.024\right)$ at 18 months. Thus, although novelty preference scores decreased with increasing delays in both groups, this decrease was more pronounced in group Neo-Hibo, which differed significantly from group Neo-C at the 10 and 120 s delays $(p<0.05)$. These group differences cannot be explained by an effect of the neonatal hippocampal lesions on viewing behaviors since the two groups did not differ in total familiarization time and total retention time at all ages and all delays ( $p>0.05$ for all).

Because there was significant individual variation among hippocampal lesion extent, we also investigated how this variation impacted the magnitude of incidental recognition memory impairment found at 18 months of age. There were no significant correlations between novelty scores and extent of total hippocampal volume damage or unintended damage for any delays at the age of 18 months $(p>0.05$ for all). 

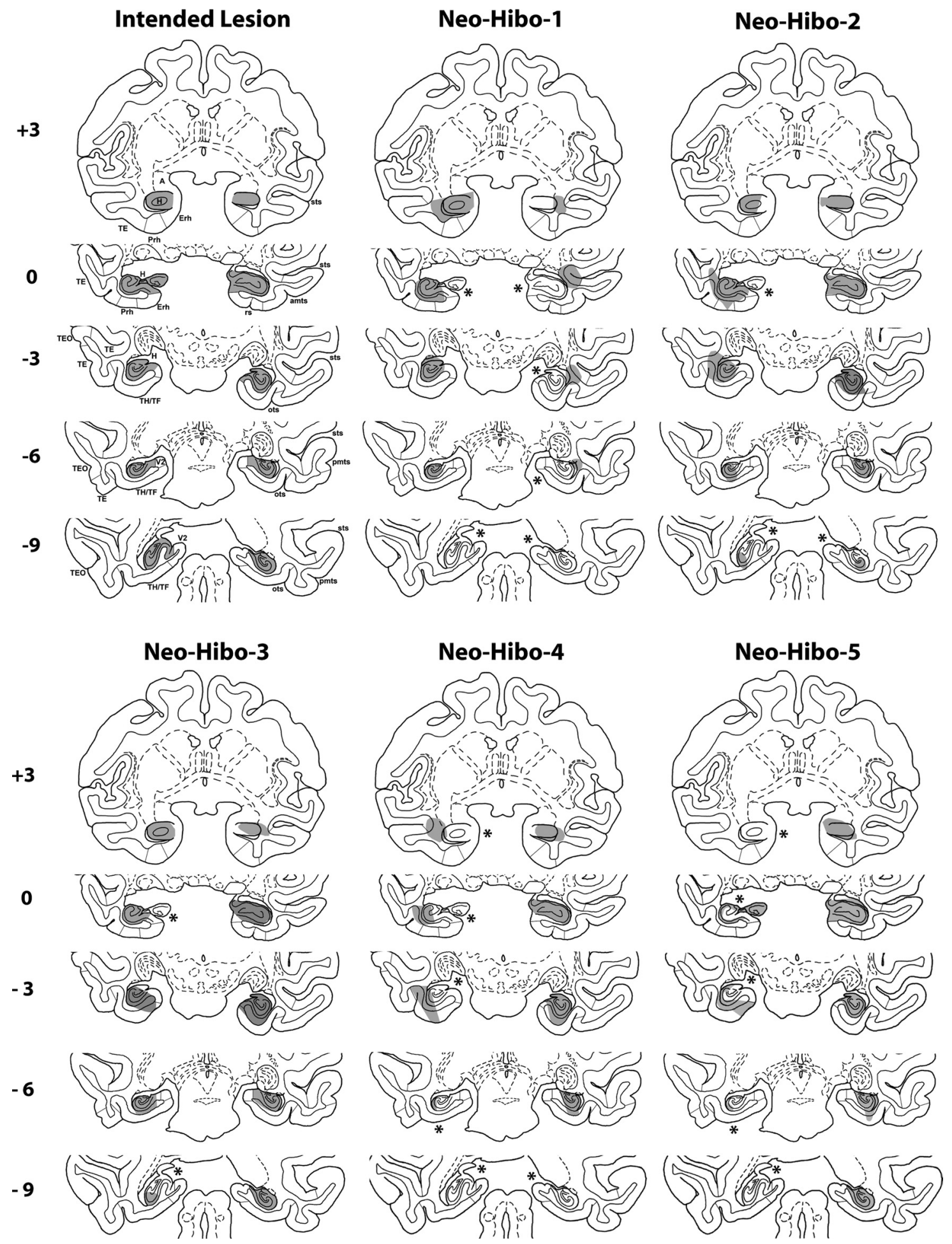

Figure 2. Each column depicts coronal sections through the hippocampal formation of a 1-week-old macaque brain. The left column depicts in gray the intended lesion as reconstructed onto five anterior-posterior (top to bottom) levels. The numerals to the left of each coronal section indicate the distance in millimeters from the interaural plane. The remaining five columns depict the extent of hypersignals seen on matched FLAIR MR images for each case in group Neo-Hibo and reconstructed onto sections of the normal macaque infant brain. * denotes levels where sparing of the hippocampus was noted. A, Amygdala; amts, anterior middle temporal sulcus; ERh, entorhinal cortex; ots, occipitotemporal sulcus; pmts, posterior middle temporal sulcus; PRh, perirhinal cortex; rs, rhinal sulcus; sts, superior temporal sulcus; TE, TEO, and TH/TF, cytoarchitectonic fields as described by von Bonin and Bailey (1947).

\section{Discussion}

\section{Normal development of recognition memory}

Infant monkeys with an intact hippocampus showed robust novelty preference as early as 1.5 months of age even at the long delays tested, and this preference became more robust by 6 months of age. These findings concur with many other reports indicating the presence of incidental object-recognition memory abilities in early infancy in monkeys (Gunderson and Sackett, 1984; Gunderson and Swartz, 1984; Bachevalier et al., 1993) as well as humans (for review, see Fagan, 1990; Pascalis and de Schonen, 1994; Rose et al., 2004). Yet, 


\section{Post-Surgical FLAIR Neo-Hibo-2}
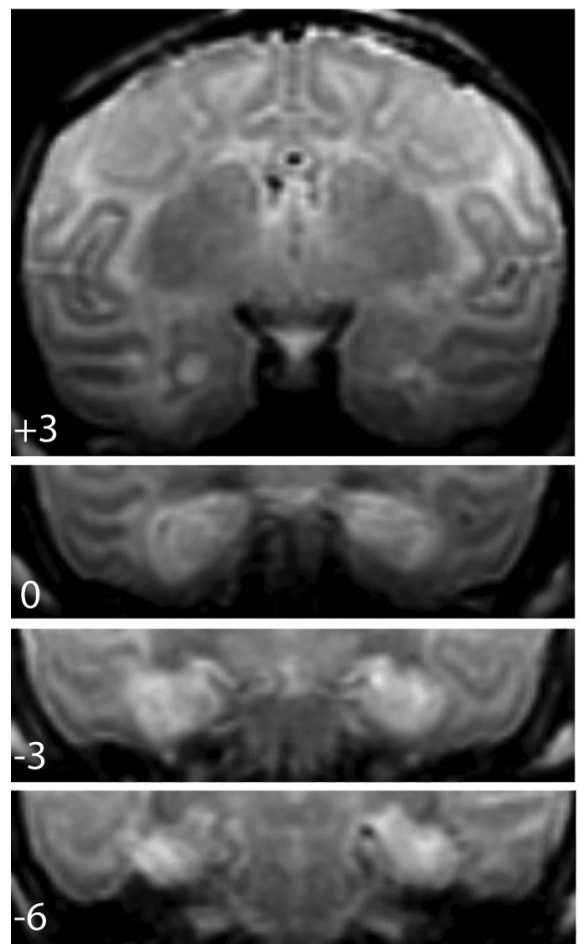

1 Year Post-Surgical MRI Neo-Hibo-2
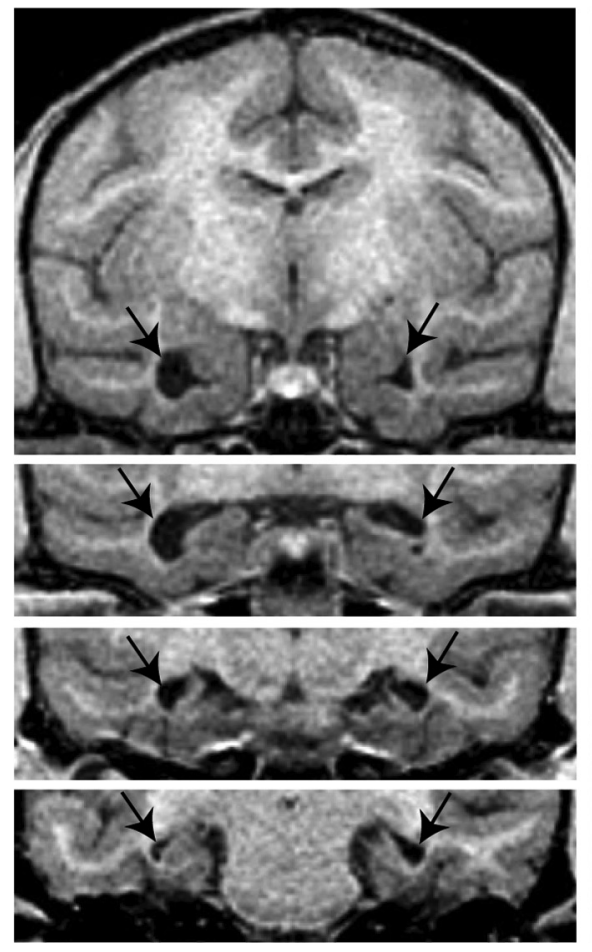

\section{Year Post-Surgical MRI Neo-C-2}
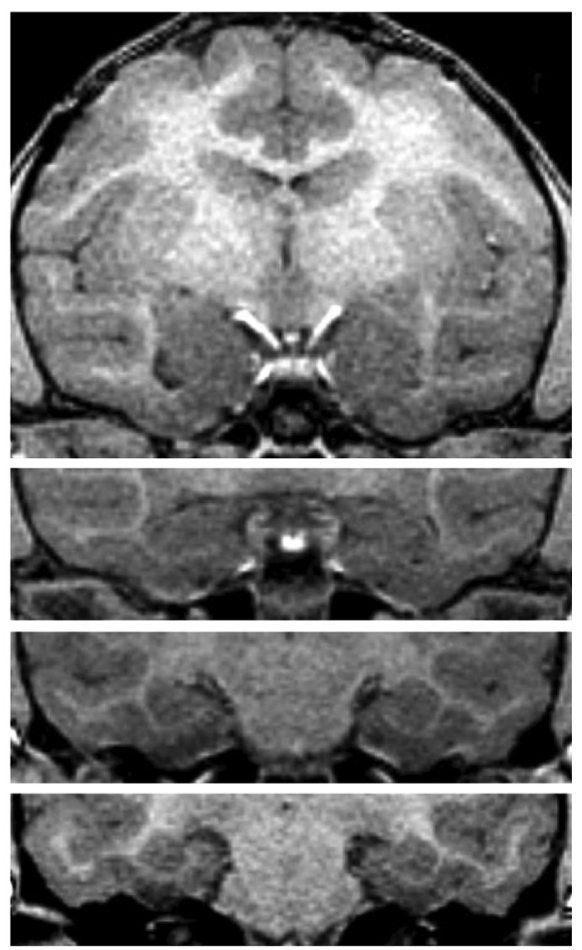

Figure 3. Coronal MRI sections through the hippocampal formation of case Neo-Hibo-2 (left and middle) and case Ne0-C-2 (right). The postsurgical FLAIR images (left) depict hypersignals (white areas) resulting from edema caused by cell death. The 1 year postsurgical structural T1 images (middle) depict amount of hippocampal volume reduction that has resulted from the neurotoxin injection (for comparison, see case Neo-C-2). Arrows indicate enlarged ventricles due to loss of hippocampal tissue.

Table 3. Individual novelty preference scores for all color trials for each subject

\begin{tabular}{|c|c|c|c|c|c|c|c|c|c|c|c|c|}
\hline & \multicolumn{4}{|c|}{ Delay at 1.5 months } & \multicolumn{4}{|c|}{ Delay at 6 months } & \multicolumn{4}{|c|}{ Delay at 18 months } \\
\hline & $10 \mathrm{~s}$ & $30 \mathrm{~s}$ & $60 \mathrm{~s}$ & $120 \mathrm{~s}$ & $10 \mathrm{~s}$ & $30 \mathrm{~s}$ & $60 \mathrm{~s}$ & $120 s$ & $10 \mathrm{~s}$ & $30 \mathrm{~s}$ & $60 \mathrm{~s}$ & $120 \mathrm{~s}$ \\
\hline \multicolumn{13}{|l|}{ Group Neo-C } \\
\hline Neo-C-1 & 68.2 & 77.4 & 60.7 & 63.9 & 82.8 & 79.1 & 83.0 & 79.1 & 72.6 & 79.5 & 77.5 & 79.2 \\
\hline $\mathrm{NeO}-\mathrm{C}-2$ & 71.5 & 64.0 & 62.4 & 66.8 & 81.4 & 76.4 & 75.6 & 79.9 & 73.5 & 74.4 & 74.0 & 68.1 \\
\hline $\mathrm{NeO}-\mathrm{C}-3$ & 56.9 & 65.5 & 73.2 & 54.1 & 67.4 & 80.8 & 69.9 & 72.9 & 74.3 & 70.5 & 69.2 & 62.0 \\
\hline $\mathrm{NeO}-\mathrm{C}-4$ & 63.0 & 65.7 & 59.6 & 62.5 & 61.7 & 69.8 & 67.1 & 75.6 & 75.6 & 69.2 & 71.9 & 65.0 \\
\hline $\mathrm{NeO}-\mathrm{C}-5$ & 63.1 & 67.7 & 73.4 & 75.0 & 76.3 & 69.6 & 71.9 & 76.1 & 73.3 & 56.1 & 61.0 & 54.5 \\
\hline $\mathrm{Ne} 0-\mathrm{C}-6$ & 58.8 & 72.6 & 65.6 & 63.1 & 60.9 & 73.8 & 55.9 & 62.1 & 72.6 & 62.5 & 59.6 & 61.8 \\
\hline Average & 63.6 & 68.8 & 65.8 & 64.2 & 71.7 & 74.9 & 70.6 & 74.3 & 73.7 & 68.7 & 68.9 & 65.1 \\
\hline SEM & 2.2 & 2.1 & 2.5 & 2.8 & 4.0 & 1.9 & 3.7 & 2.7 & 0.5 & 3.4 & 2.9 & 3.4 \\
\hline \multicolumn{13}{|l|}{ Group Neo-Hibo } \\
\hline Neo-Hibo-1 & 64.0 & 61.5 & 78.3 & 63.1 & 75.7 & 71.9 & 63.1 & 76.3 & 60.2 & 57.5 & 73.6 & 58.1 \\
\hline Neo-Hibo-2 & 59.3 & 70.7 & 63.9 & 56.1 & 71.2 & 74.8 & 68.7 & 76.6 & 78.0 & 67.2 & 76.6 & 58.1 \\
\hline Neo-Hibo-3 & 67.7 & 70.1 & 57.5 & 63.9 & 65.9 & 77.7 & 73.6 & 76.8 & 62.9 & 68.9 & 69.2 & 55.4 \\
\hline Neo-Hibo-4 & 56.1 & 59.7 & 68.0 & 65.4 & 63.7 & 71.1 & 75.4 & 69.3 & 71.1 & 63.2 & 77.8 & 55.6 \\
\hline Neo-Hibo-5 & 59.9 & 62.1 & 56.5 & 59.9 & 62.2 & 82.8 & 63.5 & 67.2 & 59.0 & 63.1 & 61.4 & 53.7 \\
\hline Average & 61.4 & 64.8 & 64.8 & 61.7 & 67.7 & 75.7 & 68.9 & 73.3 & 66.3 & 64.0 & 71.8 & 56.2 \\
\hline SEM & 2.0 & 2.3 & 4.0 & 1.7 & 2.5 & 2.1 & 2.5 & 2.1 & 3.6 & 2.0 & 3.0 & 0.9 \\
\hline
\end{tabular}

unlike the present findings, human studies using brief familiarization time showed that visual recognition over long delays increased progressively during the first year of life (Fagan, 1972; Cornell, 1974; Rose, 1981; Diamond, 1990). For example, Diamond (1990) reported that 4-month-old infants recognized stimuli after short delays of $10 \mathrm{~s}$ but not after delays of $15 \mathrm{~s}, 1 \mathrm{~min}$, and $10 \mathrm{~min}$, whereas 6-month-olds recognized them with delays of $1 \mathrm{~min}$ and 9-montholds recognized them with delays of $10 \mathrm{~min}$. It is possible that the lack of a developmental progression in the strength of visual recognition in monkeys may be due to the length of the delays that, unlike those used in human infants, did not span more than $2 \mathrm{~min}$. More importantly, the findings of the present study indicated the presence of a delay-dependent recognition effect that was apparent after 6 months of age, such that by 18 months, novelty preference was weaker at the longest delays than at the shortest delays. The only evidence that such an effect may also be present in human infants was provided by Bahrick and Pickens (1995). These authors reported that 3-monthold infants showed novelty preference at a delay of $1 \mathrm{~min}$, no preference at delays of $1 \mathrm{~d}$ and 2 weeks, and familiarity preference at longer delays of 1 and 3 months. 


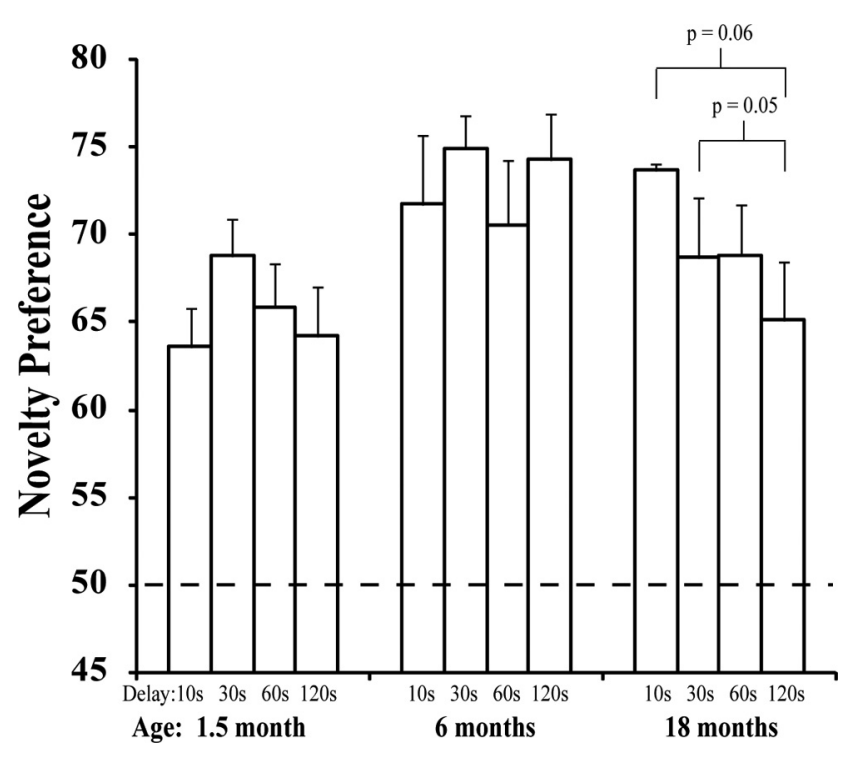

Figure 4. Mean percentage of time $( \pm S E M)$ spent viewing the novel pictures for animals with neonatal sham operations (group Neo-C) across each age and delay. Chance performance is depicted by the horizontal dashed line.

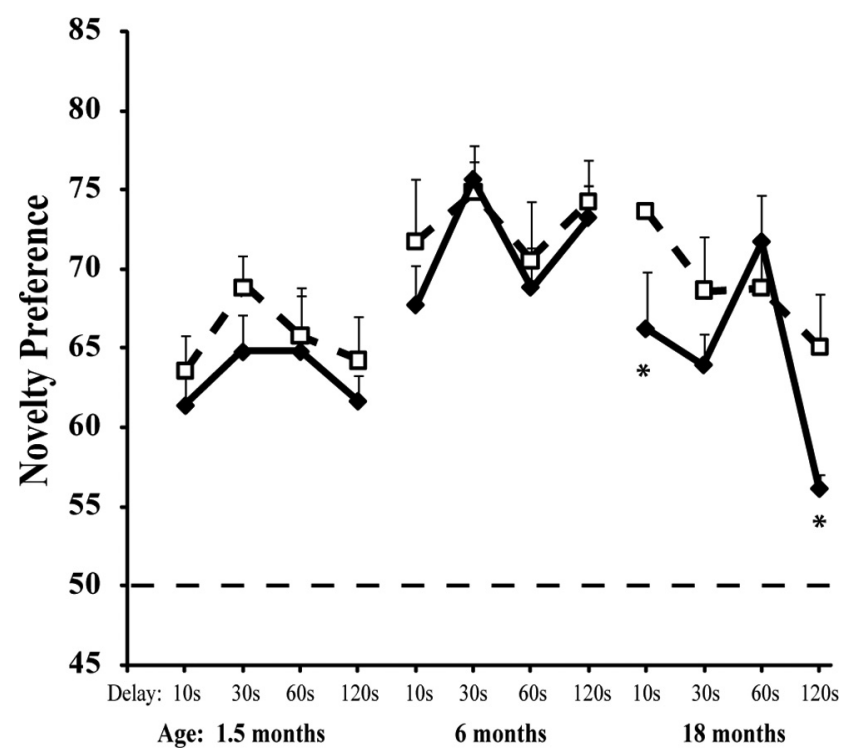

Figure 5. Mean percentage of time ( $\pm S E M)$ spent viewing the novel pictures for animals with neonatal hippocampal lesions (group Neo-Hibo, black diamond and solid line) compared with animals with neonatal sham operations (group Neo-C, open square and dashed line) across each age and delay. Chance performance is depicted by the horizontal dashed line and ${ }^{*}$ indicates group differences. $p<0.05$.

It is possible that the delay-dependent effect found at 18 months of age may simply be ascribed to the effects of rearing conditions (surrogate-nursery-peer rearing) provided to the infant monkeys given that early stress may impact the development of cognitive processes (Sánchez et al., 1998; Pryce et al., 2005). This is, however, unlikely since at 18 months of age the shamoperated animals obtained novelty preference scores $(74,69$, and $65 \%$ at the 10,60 , and 120 s delays, respectively) similar to those we obtained in adult sham-operated controls that were motherreared in a large social group using the same task, stimuli, and delays $(73,68$, and $68 \%$, respectively) (Zeamer and Bachevalier, 2005). Thus, the emergence of the delay-dependent recognition memory later in maturation suggests that the brain structures mediating these early developing recognition abilities may undergo significant modifications after 6 months of age in monkeys, as discussed below.

Recognition memory and neonatal lesion of the hippocampus Monkeys with selective neonatal hippocampal lesions performed just as well as sham-operated controls at both 1.5 and 6 months of age and did not show a recognition-memory deficit until they reached 18 months of age. The group by delay interaction at this later age demonstrated that, although for both groups novelty preference scores decreased with increasing delays, this effect was more pronounced in animals with neonatal hippocampal lesions than those with sham-operations. This pattern of impairment suggests that with maturation the animals with neonatal hippocampal lesions grow into a recognition-memory loss.

Nevertheless, despite the sharp decline in performance at the longest delay of $120 \mathrm{~s}$ in animals with neonatal hippocampal lesions at 18 months, their novelty preference scores remained above chance level, indicating that recognition memory was not totally abolished. This significant sparing of incidental recognition abilities differs from the striking lack of recognition memory reported in adult monkeys with selective hippocampal lesions when delays were increased to $30 \mathrm{~s}$ and longer (Nemanic et al., 2004). Although it is not yet known whether recognitionmemory performance of animals with neonatal hippocampal lesions will continue to decline as they will reach adulthood ( 5 years in monkeys), the sparing of function at the early ages suggests that brain structures other than the hippocampus may be able to support incidental recognition-memory processes even when delays up to $2 \mathrm{~min}$ are used.

\section{Neural substrates of incidental recognition memory in infancy}

Both the emergence of delay-dependent recognition-memory performance at 18 months of age in the sham-operated animals and the recognition-memory impairment observed after neonatal hippocampal lesions at that same age suggest that important maturational changes in the neural substrate supporting incidental recognition memory occurred after 6 months of age in monkeys. These developmental trajectory patterns in both groups suggest that the medial temporal cortical structures, such as the perirhinal and parahippocampal cortex, known to mediate this function in adulthood (Buffalo et al., 1999; Nemanic et al., 2004), could support incidental recognition-memory processes in which the hippocampus will ultimately participate. This proposal concurs with our current knowledge of the postnatal maturation of the medial temporal lobe structures in monkeys.

Detailed descriptions of the neurobiological development of structures within the medial temporal lobe are still lacking. Nevertheless, available data suggest that the medial temporal cortical areas seem to mature earlier than the hippocampus (for review, see Alvarado and Bachevalier, 2000; Zeamer et al., 2009). Thus, most of the neurogenesis in medial temporal cortical areas occurs prenatally, whereas several morphological and neurochemical changes occur within the first few months postnatally (Webster et al., 1991, 1995; Berger and Alvarez, 1994). By contrast, the basic trisynaptic pathway of the hippocampus (ERh $\rightarrow$ dentate gyrus $\rightarrow$ $\mathrm{CA} 3 \rightarrow \mathrm{CA} 2 \rightarrow \mathrm{CA} 1 \rightarrow$ subiculum) exhibits a protracted postnatal maturation that extends until the beginning of the second postnatal year (Seress and Ribak, 1995a,b; Lavenex et al., 2007). This delayed postnatal maturation is substantiated by an overall increase in hippocampal volume from birth to 11 months of age in monkeys, as revealed in a recent longitudinal structural neu- 
roimaging study (Payne et al., 2009). Finally, whereas both cholinergic and GABAergic neurotransmitter systems within the hippocampus are present at birth, they undergo considerable modifications postnatally (Lavenex et al., 2007). Given the different developmental trajectories between the temporal cortical areas and the hippocampus, it is likely that early in development, when the hippocampal formation may not be fully functional, the surrounding cortical areas could support incidental recognition memory processes even at longer delays. However, between 6 and 18 months of age, as the hippocampus approaches functional maturity, it may begin to more fully interact with the cortical areas to support this function. If this interpretation is correct, one would expect that, unlike neonatal hippocampal lesions, damage to the perirhinal cortex in infancy would have deleterious effects on incidental memory processes at early ages. Our preliminary results are consistent with this prediction. Neonatal perirhinal lesions in monkeys altered novelty preference at all ages tested $(1.5,6$, and 18 months) and this deficit became more profound as the animals matured (Zeamer and Bachevalier, 2009). Thus, the neurobiological evidence together with the present behavioral findings indicate the emergence of significant functional interactions between the medial temporal cortical areas and the hippocampus (Pihlajamäki et al., 2004; VanElzakker et al., 2008; Albasser et al., 2010) after 6 months of age and suggest that incidental recognition memory processes appear to be more widely distributed in the immature brain but become more refined as development progresses (Webster et al., 1995).

\section{Implications for the development of incidental recognition memory in humans}

The present findings demonstrate that recognition-memory processes in monkeys are available in early infancy, although the medial temporal lobe structures that support this function undergo significant modifications as the animals mature. These findings have at least two significant implications. First, they substantiate mounting evidence that, in both humans and monkeys (Goldman and Rosvold, 1972; Webster et al., 1995; Bates, 2004; Zeamer et al., 2009), the early developing brain may use different neural pathways to support the same cognitive functions described in adults. Second, they could help explain findings from recent reports of developmental amnesia in humans. As recently reviewed (de Haan et al., 2006), patients suffering from developmental amnesia due to early postnatal hippocampal damage show profound deficits in recall but relatively intact recognition memory. The authors concluded that the intact recognition ability in these cases could reflect either intact "familiarity-based recognition" processes known to be mediated by the medial temporal cortical areas (Mishkin et al., 1997; Brown and Aggleton, 2001; O'Reilly and Rudy, 2001; Yonelinas, 2002; Mayes et al., 2003) or greater plasticity in the medial temporal cortical areas during development (Webster et al., 1991, 1995) that could take over some of the recognition-memory processes normally mediated by the hippocampus. The present findings are congruent with both proposals since not only did we find that the medial temporal cortical areas are sufficient to mediate incidental recognition memory before this function necessitated the critical interactions of the temporal cortical areas and the hippocampus later in development, but also that the same cortical areas appeared to take over some of the hippocampal-dependent recognition memory processes in the absence of a functional hippocampus, since novelty preference was not totally abolished at any delay after neonatal hippocampal lesions as it was in the adults (Zola et al., 2000; Nemanic et al., 2004). However, given that in the cases of human developmental amnesia, memory difficulties did not become apparent until the children reached school age (Vargha-Khadem et al., 2003), it is still possible that the sparing of incidental recognition memory observed at 18 months of age may fade away. Thus, follow-up of the animals with neonatal hippocampal lesions as they reach adulthood is clearly needed to demonstrate whether they will grow into a more severe recognition memory loss.

\section{References}

Albasser MM, Poirier GL, Aggleton JP (2010) Qualitatively different modes of perirhinal-hippocampal engagement when rats explore novel vs. familiar objects as revealed by c-Fos imaging. Eur J Neurosci 31:134-147.

Alvarado MC, Bachevalier J (2000) Revisiting the maturation of medial temporal lobe memory functions in primates. Learn Mem 7:244-256.

Bachevalier J, Brickson M, Hagger C (1993) Limbic-dependent recognition memory in monkeys develops early in infancy. Neuroreport 4:77-80.

Bahrick LE, Pickens JN (1995) Infant memory for object motion across a period of three months: implications for a four-phase functions. J Exp Child Psych 59:343-371.

Bates EA (2004) Explaining and interpreting deficits in language development across clinical groups: where do we go from here? Brain Lang $88: 248-253$.

Berger B, Alvarez C (1994) Neurochemical development of the hippocampal region in the fetal rhesus monkey. II. Immunocytochemistry of peptides, calcium-binding proteins, DARPP-32, and monoamine innervation in the entorhinal cortex by the end of gestation. Hippocampus 4:85-114

Brown MW, Aggleton JP (2001) Recognition memory: what are the roles of the perirhinal cortex and hippocampus? Nat Rev Neurosci 2:51-61.

Buffalo EA, Ramus SJ, Clark RE, Teng E, Squire LR, Zola SM (1999) Dissociation between the effects of damage to perirhinal cortex and area TE. Learn Mem 6:572-599.

Cornell EH (1974) Infants' discrimination of photographs of faces following redundant presentations. J Exp Child Psychol 18:98-106.

de Haan M, Mishkin M, Baldeweg T, Vargha-Khadem F (2006) Human memory development and its dysfunction after hippocampal injury. Trends Neurosci 29:374-381.

Diamond A (1990) Rate of maturation of the hippocampus and the developmental progression of children's performance on the delayed nonmatching to sample and visual paired comparison tasks. Ann N Y Acad Sci 608:394-433.

Fagan JF 3rd (1970) Memory in the infant. J Exp Child Psychol 9:217-226.

Fagan JF 3rd (1972) Infants' recognition memory for faces. J Exp Child Psychol 14:453-476.

Fagan JF 3rd (1990) The paired-comparison paradigm and infant intelligence. Ann N Y Acad Sci 608:337-364.

Goldman PS, Rosvold HE (1972) The effects of selective caudate lesions in infant and juvenile rhesus monkeys. Brain Res 43:53-66.

Goursaud AP, Bachevalier J (2007) Social attachment in juvenile monkeys with neonatal lesion of the hippocampus, amygdala and orbital frontal cortex. Behav Brain Res 176:75-93.

Gunderson VM, Sackett GP (1984) Development of pattern recognition in infant pigtailed macaques (Macaca nemestrina). Dev Psych 20:418-426.

Gunderson VM, Swartz KB (1985) Visual recognition in infant pigtailed macaques after a 24-hour delay. Am J Prim 8:259-264.

Lavenex P, Banta Lavenex P, Amaral DG (2007) Postnatal development of the primate hippocampal formation. Dev Neurobio 29:179-192.

Málková L, Bachevalier J, Mishkin M, Saunders RC (2001) Neurotoxic lesions of perirhinal cortex impair visual recognition memory in rhesus monkeys. Neuroreport 12:1913-1917.

Mayes AR, Holdstock JS, Isaac CL, Hunkin NM, Roberts N (2002) Relative sparing of item recognition memory in a patient with adult-onset damage limited to the hippocampus. Hippocampus 12:325-340.

McKee RD, Squire LR (1993) On the development of declarative memory. J Exp Psychol Learn Mem Cogn 19:397-404.

Mishkin M, Suzuki WA, Gadian DG, Vargha-Khadem F (1997) Hierarchical organization of cognitive memory. Philos Trans R Soc Lond B Biol Sci 352:1461-1467.

Nelson CA (1995) The ontogeny of human memory: a cognitive neuroscience perspective. Dev Psychol 31:723-738.

Nelson CA, Webb SJ (2003) A cognitive neuroscience perspective on early memory development. In: The cognitive neuroscience of development (de Hann M, Johnson MH, eds), pp. 99-125. Psychology. 
Nemanic S, Alvarado MC, Price RE, Jackson EF, Bachevalier J (2002) Assessment of locus and extent of neurotoxic lesions in monkeys using neuroimaging techniques: a replication. J Neurosci Methods 121:199_ 209.

Nemanic S, Alvarado MC, Bachevalier J (2004) The hippocampal/ parahippocampal regions and recognition memory: insights from visual paired comparison versus object-delayed nonmatching in monkeys. J Neurosci 24:2013-2026.

O'Reilly RC, Rudy JW (2001) Conjunctive representations in learning and memory: principles of cortical and hippocampal function. Psychol Rev 108:311-345

Pascalis O, Bachevalier J (1999) Neonatal aspiration lesions of the hippocampal formation impair visual recognition memory when assessed by paired-comparison task but not by delayed nonmatching-to-sample task. Hippocampus 9:609-616.

Pascalis O, de Schonen S (1994) Recognition memory in 3- to 4-day-old human neonates. Neuroreport 5:1721-1724.

Pascalis O, de Haan M, Nelson CA, de Schonen S (1998) Long-term recognition memory for faces assessed by visual paired comparison in 3- and 6-month old infants. J Exp Psychol Learn Mem Cogn 24:249-260.

Pascalis O, Hunkin NM, Holdstock JS, Isaac CL, Mayes AR (2004) Visual paired comparison performance is impaired in a patient with selective hippocampal lesions and relatively intact item recognition. Neuropsychologia 42:1293-1300.

Payne C, Machado CJ, Bliwise NG, Bachevalier J (2009) Maturation of the hippocampal formation and amygdala in Macaca mulatta: a volumetric magnetic resonance imaging study. Hippocampus. Advance online publication. Retrieved December 1, 2009. doi:10.1002/hipo.20688.

Pedhazur E J (1982) Multiple regression in behavioral research: explanation and prediction. (2nd ed.) New York: Holt, Rinehart and Winston.

Pihlajamäki M, Tanila H, Könönen M, Hänninen T, Hämäläinen A, Soininen H, Aronen HJ (2004) Visual presentation of novel objects and new spatial arrangements of objects differentially activates the medial temporal lobe subareas in humans. Eur J Neurosci 19:1939-1949.

Pryce CR, Rüedi-Bettschen D, Dettling AC, Weston A, Russig H, Ferger B, Feldon J (2005) Long-term effects of early-life environmental manipulations in rodents and primates: potential animal models of depression. Neurosci Biobehav Rev 29:649-674.

Resende M, Chlan-Fourney J, Bachevalier J (2002) Neonatal neurotoxic lesions of the hippocampal formation do not impair recognition memory in infant macaques. Soc Neurosci Abstr 28:183.2.

Rose SA (1981) Developmental changes in infants' retention of visual stimuli. Child Dev 52:227-233.

Rose SA, Feldman JF, Jankowski JJ (2004) Infant visual recognition memory. Dev Rev 24:74-100.
Sackett GP, Ruppenthal GC, Davis AE (2002) Survival, growth, health, and reproduction following nursery rearing compared with mother rearing in pigtailed monkeys (Macaca nemestrina). Am J Primatol 56:165-183.

Sánchez MM, Hearn EF, Do D, Rilling JK, Herndon JG (1998) Differential rearing affects corpus callosum size and cognitive function of rhesus monkeys. Brain Res 812:38-49.

Seress L, Ribak CE (1995a) Postnatal development of CA3 pyramidal neurons and their afferents in the Ammon's horn of Rhesus monkeys. Hippocampus 5:217-231.

Seress L, Ribak CE (1995b) Postnatal development and synaptic connections of hilar mossy cells in the hippocampal dentate gyrus of rhesus monkeys. J Comp Neurol 355:93-110.

VanElzakker M, Fevurly RD, Breindel T, Spencer RL (2008) Environmenta novelty is associated with a selective increase in Fos expression in the output elements of the hippocampal formation and perirhinal cortex. Learn Mem 15:899-908.

Vargha-Khadem F, Salmond CH, Watkins KE, Friston KJ, Gadian DG, Mish kin M (2003) Developmental amnesia: effect of age at injury. Proc Nat Acad Sci U S A 100:10055-10060.

von Bonin G, Bailey P (1947) The neocortex of Macaca mulatta. Urbana, IL: University of Illinois.

Webster MJ, Ungerleider LG, Bachevalier J (1991) Connections of inferior temporal areas TE and TEO with medial temporal-lobe structures in infant and adult monkeys. J Neurosci 11:1095-1116.

Webster MJ, Ungerleider LG, Bachevalier J (1995) Development and plasticity of the neural circuitry underlying visual recognition memory. Can J Physiol Pharmacol 73:1364-1371.

Yonelinas AP (2002) The nature of recollection and familiarity: a review of 30 years of research. J Mem Lang 46:441-517.

Zeamer AE, Bachevalier J (2005) Visual recognition memory and the hippocampus in monkeys: effects of parameter manipulations in the VPC task. Soc Neurosci Abstr 31:195.8.

Zeamer AE, Bachevalier J (2009) Neonatal lesions of the perirhinal cortex alter the development of object recognition memory abilities in monkeys. Soc Neurosci Abstr 35:98.6.

Zeamer AE, Resende M, Heuer E, Bachevalier J (2006) The development of infant monkeys' recognition memory abilities in the absence of a functional hippocampus. Soc Neurosci Abstr 32:718.3.

Zeamer AE, Alvarado MC, Bachevalier J (2009) Development of media temporal lobe memory processes in non-human primates. In: Oxford handbook of developmental behavioral neuroscience (Blumberg M, Freeman J, Robinson S, eds), pp. 607-616. New York: Oxford UP.

Zola SM, Squire LR, Teng E, Stefanacci L, Buffalo EA, Clark RE (2000) Impaired recognition memory in monkeys after damage limited to the hippocampal region. J Neurosci 20:451-463. 